Entropy 2004, 6, 38-49

\title{
Entropy
}

ISSN 1099-4300

www.mdpi.org/entropy/

Full Paper

\section{The Decrease in Entropy via Fluctuations}

\section{Lyndsay G M Gordon}

11 Guildford Tce., Thorndon, Wellington, New Zealand. E-mail: lgordon@actrix.gen.nz

Received: 29 August 2003 / Accepted: 17 December 2003/ Published: 22 March 2004

\begin{abstract}
Classical and quantum aspects of fluctuations are reviewed in a discussion on the universality of the second law. Consideration is given to the need of information and the requirement of a ratchet and pawl-type mechanism for the utilization of energy from fluctuations. Some aspects of the quantum theory of the Copenhagen school are compared to those of Bohm within the discussion.
\end{abstract}

Keywords: Szilard, ratchets, second law, Maxwell's demon, quantum theory, Bohm

\section{Introduction}

In his paper "On the decrease in entropy in a thermodynamic system by the intervention of intelligent beings", Szilard [1] devised a method for linking mechanics and thermodynamics in a process associated with fluctuations. This article has been referenced many times, both by those who have built on the foundations laid by Szilard and by those who have denied his major tenet, that information gathering is essential in the cycling nature of the Szilard machine during the process of decreasing entropy. 
Why are there two schools of thought and how may their differences be addressed? Although Szilard's paper was written in the aftermath of the major advances in quantum mechanics, it shows no reference to this field. However more recently, the problem has been extended to give it a quantum mechanical aspect that has brought information as a thermodynamic property further into contention. In an attempt to resolve these differences, an experimental method is outlined below. Whether such technology is currently available for such a project is a point to be answered.

\section{The Classical Arguments}

We shall begin the discussion with a review of Szilard's device and Popper's refutation. The device of Szilard [1] is expanded into a cycle of 4 stages (A to D, Fig. 1) so that it is comparable with the more advanced devices to be described later in the present paper. An intelligent being couples the Szilard device to the load after obtaining and using knowledge of the gas molecule's position relative to the piston. Not only did Szilard conceive of the possibility that information gained by the intelligent being could be used for the reduction of the entropy of the system, but reasoned

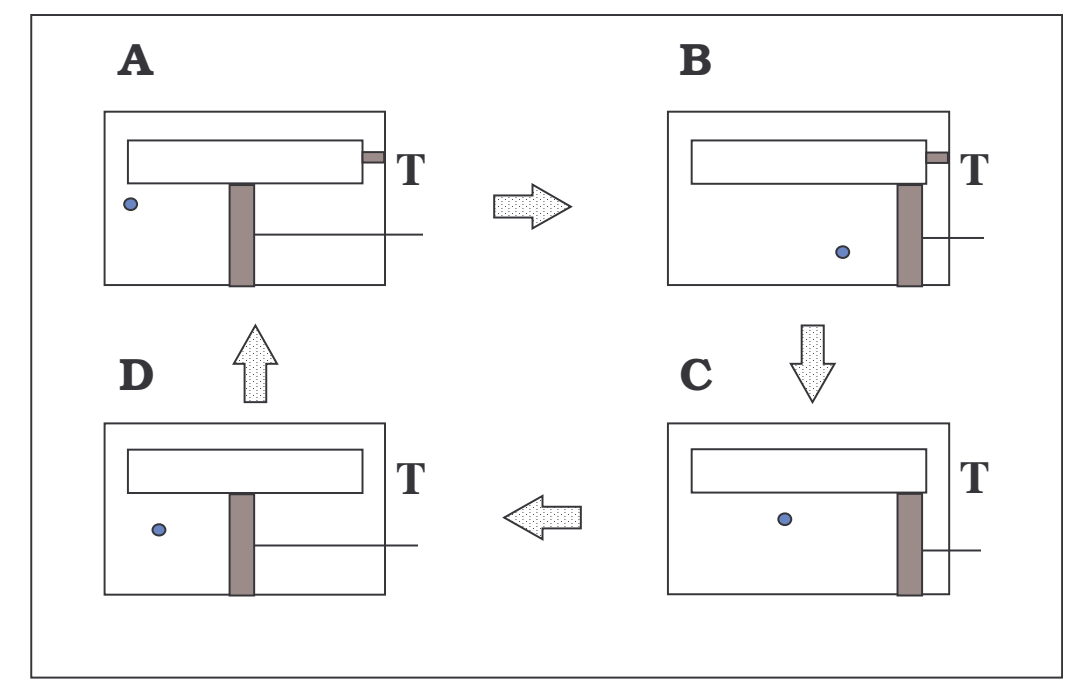

FIGURE 1. The device of Szilard. (A) The load has been coupled to the piston by an intelligent being and simultaneously the trapdoor has been closed. (B) Work has been accomplished by the gas expansion. (C) The trapdoor has been opened and simultaneously the load has been uncoupled and supported. (D) The piston has been moved to a central position. The cycle recommences at A with the gas molecule on the same or opposite side of the piston. 
controversially that that information was essential. The being was required to operate each phase of the cycle (Fig. 1), but only one phase needed an act of 'intelligence' - the correct coupling of the load at the beginning of phase A. In its most flexible state, the system would move through its various phases so long as the being always (i) simultaneously uncoupled and locked the load when the trapdoor opened, (ii) simultaneously coupled and unlocked the load when the trapdoor closed after an observation of the gas molecule and (iii) repositioned the piston during the open state of the trapdoor.

The major quest of Szilard's was to show that the energy expended in the observation (information gathering or measurement, Fig.1A) was at least as large as the equivalent decrease in entropy produced by a completed cycle of the device.

Set against this background and guided by his anti-subjectivist belief, Popper [2] showed how the Szilard device could be modified to avoid the intelligent choice of the coupling that was required by the being. Popper concluded that both the forward and reverse motions of the piston could do either separate pieces of work or alternatively one piece of work where the Szilard device was fitted

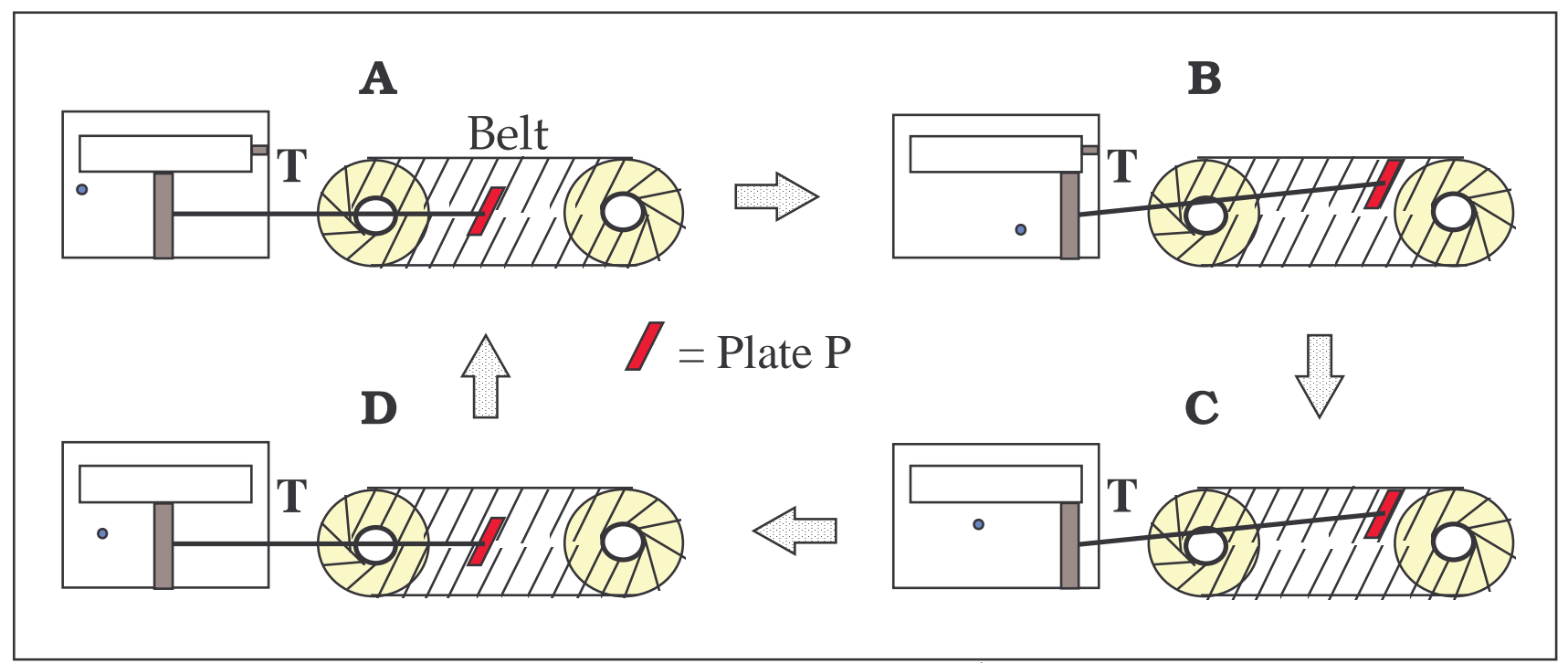

FIGURE 2. The device of Popper as perceived by the author ${ }^{1}$. The same operations as in Szilard's device (Fig. 1) except a converter is added to replace the intelligent being. The Plate P attached to the piston rod pushes or pulls the plates fixed to the converter belt. (See the text for details.)

\footnotetext{
${ }^{1}$ Presented at the International Congress of Physics, Sydney, 1980. Another conception of the gear has been given by Rothstein [3].
} 
"with some gear so that it lifts a weight in either case, without our having to know which of these two possible directions the impending movement will take". Figure 2 illustrates the author's perception of Popper's device in its four stages of a cycle corresponding to those in Figure 1.

The gear shown in Fig. 2 has two wheels around which there is a continuous belt. Plates are attached to the belt at a fixed angle and are pushed or pulled by a plate $(\mathrm{P})$ that is attached to the piston rod. $\mathrm{P}$ can slide over the other plates frictionlessly when moving between the lower and upper parts of the belt. The piston rod has frictionless joints at both ends to provide $\mathrm{P}$ with flexibility of angle. At the closing of the trapdoor the piston is free to move and as a consequence, $\mathrm{P}$ slides into the correct position to push an upper plate or to pull a lower plate. The course of this action is dependent on the location of the gas molecule. No intelligent being is required.

Szilard considered that the universality of the second law would have been in an "ominous" state if it were not for the essential payment for information that he described in his model. Popper disagreed with this analysis and showed that if there is a problem concerning the universality of the second law then it must lie elsewhere, since the information gathered by the intelligent being can be replaced by a mechanical equivalent.

Clearly, the mechanisms of Szilard and Popper are far too simple to describe the work done by a succession of thermal fluctuations. Their descriptions contain supplementary activities that have not been accounted for except for the process of locating the gas molecule. The trapdoor needs to be opened and shut, the load has to be coupled and uncoupled in a suitable manner which involves locking and unlocking of the load and the piston has to be re-centred (or repositioned) during each cycle. Because of these extra activities one can sympathize with Popper's statement that his model could not "be used to refute the second law of thermodynamics" even though he had refuted the argument of Szilard, that energy had to be expended to locate the gas molecule. Popper's conclusion appears to be based on the fact that his device could not extend beyond one fluctuation without some intervention to maintain a continuous activity for perpetual motion.

Like Popper, Feynman et al. [4] saw that the challenge to the universality of the second law lay not in the gathering of information but in the extra activities, (i) to (iii) referred to above. Unlike Popper however, Feynman argued for the futility of challenges to the second law and introduced a 
ratchet and pawl into the design to illustrate his point. Feynman saw that the ratchet and pawl were essential for moving the load unidirectionally over a long period of many fluctuations (or cycles). He concluded from his construction however, that microscopic pawls would move freely and randomly and thereby rendered useless. Here, it would seem, lies the true nub of the difficulty in classical terms. However, Feynman's model is not representative of all pawl and ratchet types. The following construction illustrates how a microscopic pawl can maintain the unidirectionality of the load in microscopic machines and yet retain perfect freedom of random movement.

Firstly, a connecting wheel (Fig. 3) with cogs (not shown) can be fixed concentrically to one of the wheels of the 'gear', originally illustrated in Fig. 2. The trapdoor in the third model is intimately linked with the pawl with which it shares the same axle (Figure 4,). When the trapdoor is open, the pawl engages a cog on the additional wheel so that it becomes non-rotatable and the load and the piston, as a consequence, become immobile in both directions. The closed trapdoor (the disengaged pawl) allows the 'gear' and the load to move freely, again in both directions. This composite mechanism associated with the Szilard engine is a converter of bi-directional to unidirectional motion of a continuous kind. The pawl fluctuates freely but its association with the trapdoor provides a perfect control over the working of the machine.

In a macroscopic machine, the pawl's position is governed by the movement of the piston. This is the basis of Feynman's ratchet and pawl device but not of the converter of Figure 3.

The labels, A to D of the diagrams in Fig. 3 correspond to the diagrams with the same labels in the Szilard and Popper machines described in Fig's 1 and 2. The trapdoor / pawl arrangement can be likened to a unimolecular equilibrium where the molecule fluctuates between its two states (pawl engaged / disengaged) in which it can have different average resident times. Feynman's conditions are hereby satisfied. The gas molecule, during the open state of the trapdoor, is free to move from one side of the piston to the other or remain on the same side. The side it takes during the closing of the trapdoor determines the new direction of the piston and this in turn determines the new position of the plate, $\mathrm{P}$, on the belt. In either chamber, the molecular gas expands to lift the load.

Unlike the Szilard device, the machine of Figure 3 does not need an intelligent being for its operation, nor does it require external aid to perform the extra activities, as does Popper's device. The microscopic ratchet and pawl behave in the expected manner to reduce the entropy of the system at the expense of heat. 


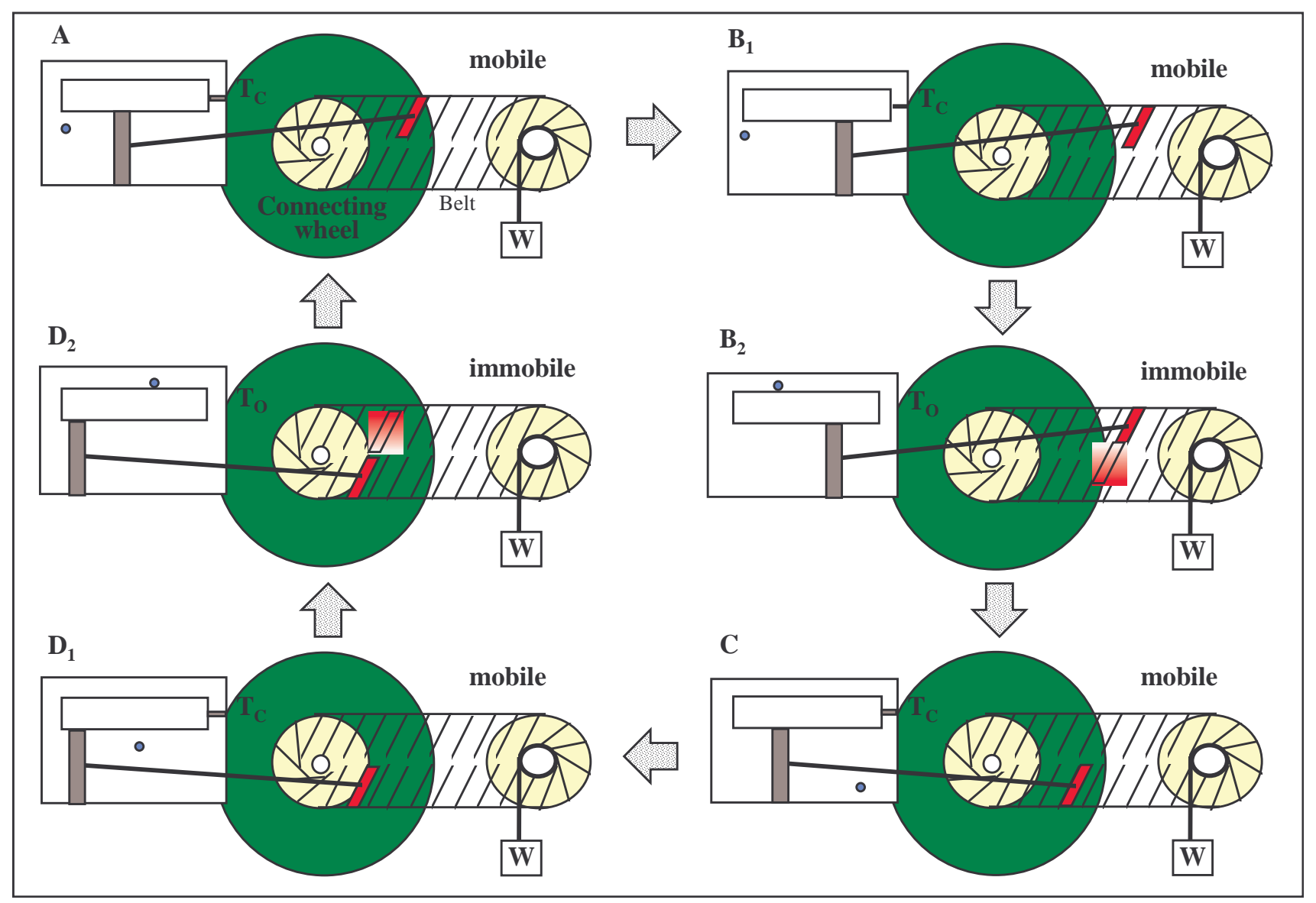

FIGURE 3. A device as described in Fig. 2 but with an additional wheel that is fixed to the axle of a converter wheel and makes a second linkage (one of control via a pawl and trapdoor) between the converter and the Szilard engine. The first linkage is via the piston rod.

FIGURE 3A. The machine is in operation with the trapdoor closed $\left(\mathrm{T}_{\mathrm{c}}\right)$ and with the pawl (not shown) disengaged. One of the belt-plates is being pushed.

FIGURE $3 B_{1}$ The trapdoor is about to open.

FIGURE 3B $\mathbf{B}_{2}$ The trapdoor is open $\left(\mathrm{T}_{\mathrm{o}}\right)$. The pawl is engaged with the connecting cogwheel. The Plate, $\mathrm{P}$, moves randomly within the confines of the immobile plates of the belt of the gear. (Two of the many possible positions of $\mathrm{P}$ are shown).

FIGURE 3C The trapdoor is closed $\left(\mathrm{T}_{\mathrm{c}}\right)$ and the pawl is disengaged. The force on the plate, $\mathrm{P}$, drives it to the lower belt where it begins to pull.

FIGURE 3D $_{1}$ The trapdoor is about to open.

FIGURE 3D $_{\mathbf{2}}$ Same as FIGURE $\mathbf{3 B}_{\mathbf{2}}$ 


\section{Plan View of $B_{2}$ (trapdoor open)}

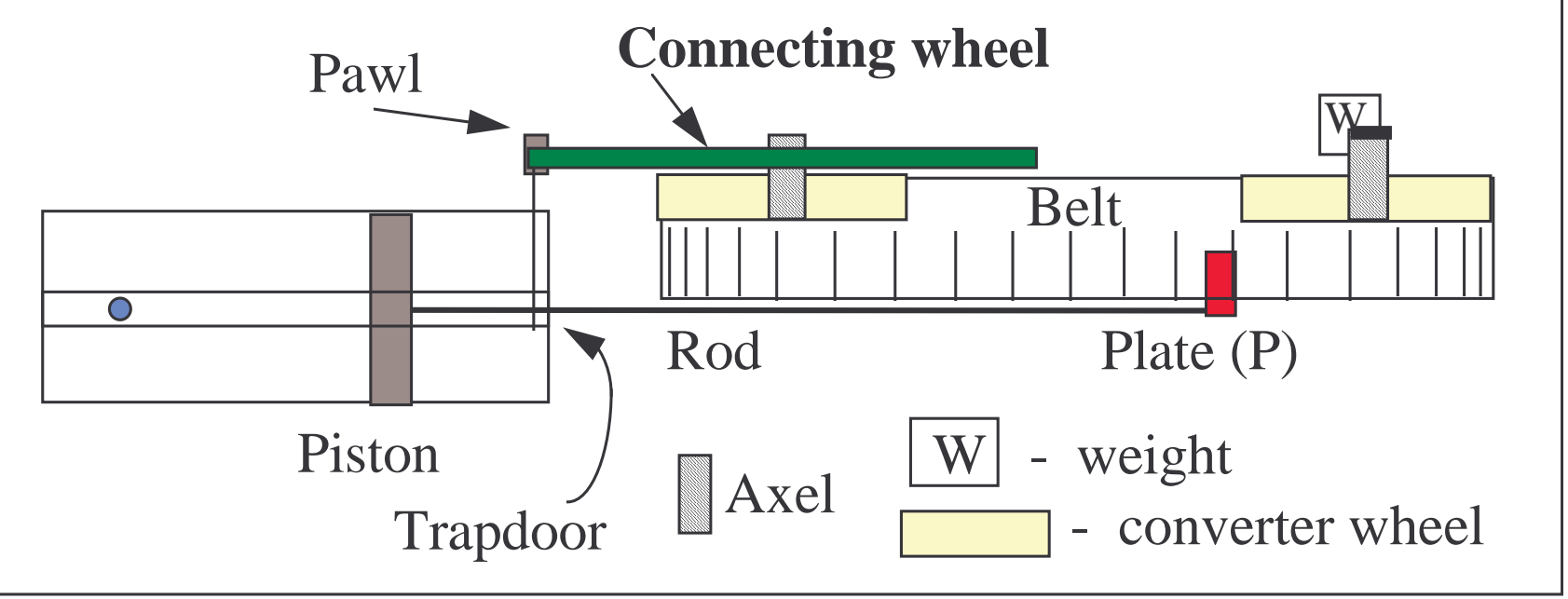

FIGURE 4. Plan view of the device illustrated in Fig. 3. The pawl and the trapdoor are fixed to the same axle lying vertically in the plane of the paper. The pawl is engaged, the trapdoor is open (not shown) at indicated position.

Within the classical domain, the analysis of the machine, illustrated in Figure 3, suggests that the second law is not universally valid. Are there other elements that need to be reviewed which may invalidate this conclusion? Indeed, the present study of individual events would be unfinished if quantum mechanical aspects were to be omitted. In the next section, the role of quantum theory will be examined.

\section{The Quantum Mechanical Argument.}

The discussion here will be confined to two different approaches to a theoretical analysis of a one-molecule gas in the Szilard-type engine, of which three have been described above. The critical process of the closing of the trapdoor is examined within the philosophical interpretations of the orthodox or Copenhagen school and of the deterministic view of Bohm. 


\section{The Copenhagen Interpretation:}

Immediately after the closing of the trapdoor in the Szilard experiment, the classical gas molecule would be located on one side or the other of the piston. In contrast, the Copenhagen School interprets the particle as a wave and so at the closure of the trapdoor, the location of the gas molecule should be found on "both" sides [5]. Obviously, this would prevent the development of a pressure gradient across the piston. Does this mean that the Szilard-type engine is doomed to everlasting inaction? No, the Copenhagen school allows the system to revert to the situation where the gas molecule is again contained completely in one of the compartments but only after the intervention by an external measuring device. This device ascertains the location of the particle and as a consequence the "collapse of the wave function" is triggered.

As in the classical Szilard engine, where the gathering of information about the gas molecule's position required at least as much energy as the engine could produce, the measurement in the quantum mechanical case ensures the same outcome whether it is due to the measurement itself or the resetting of the instrument. On this basis, information gathering has come to the fore again but not for the same reason as put forward by Szilard. With the device of Fig.3 we are not required to ascertain the position of the gas molecule (i.e. information is not required) so the essential role of the "measuring device" is simply to collapse the wave function and not to measure!

As the gas molecule is considered to be on both sides of the piston after the closing of the trapdoor and before the measurement is made, Popper's additional gear would be ineffective within this interpretation of events. However, the Copenhagen interpretation is tested by the following variation of the engine in Fig. 3.

In his autobiography, Einstein [6] recalled the artificial distinction that was made between particles suspended in a liquid and a substance that was dissolved therein. According to classical thermodynamics of the 19th century, small particles would not be able to produce an osmotic pressure, whereas dissolved substances could. The distinction was based on the arbitrary fact that we can observe suspended particles but we cannot observe the molecules in solution. The experiments of Perrin [7] terminated this fallacy. Surprisingly, similar studies could provide an investigative tool in quantum theory.

By filling the chambers of the engine in Fig. 3 with a liquid into which a visible particle is suspended and by converting the solid nature of the piston into a semi-permeable membrane, 
quantum mechanics can be studied simultaneously with the second law. No doubt, the wave function for the fluid (particle and liquid) would be more complex than that for the one-molecule gas. Nevertheless, when the trapdoor closes, the particle, according to the orthodox treatment should be on both sides of the membrane. The particle, being visible, suggests that the "uncollapsed" and "collapsed" states of the system can be continuously monitored by eye.

However, it may be considered that the continuous monitoring of the system by eye may of itself collapse the wave function immediately at the closing of the trapdoor and thereby allow an osmotic flow and a subsequent movement of the membrane (piston). [The opening of the box to view Schroedinger's cat was considered to have collapsed the wave function.] This suggests that when the eye is focused on the particle, the membrane will move backwards and forwards over time and lift the weight contrary to the second law, but remain stationary when not observed in accordance with the orthodox quantum theory. On the other hand the membrane may be found to move independently of observation in a classical manner and thus refute the Copenhagen interpretation.

Thus, the orthodox quantum theory either leads to an observable collapsed state or alternatively is disqualified from the discussion of the universality of the second law.

\section{The Bohm Interpretation:}

The quantum theory of Bohm [8] is an argument for a deterministic view of molecular events and when applied to the machine described in Figure 3 would provide an identical result to that given by classical concepts. That is, the universality of the second law is challenged and that quantum mechanics here does not provide a circumvention.

\section{Computational Science}

Many advances in human thought have been accomplished by the transference of ideas between fields of endeavour, sometimes referred to as cross-fertilization. Usually, the two fields have a common basis and in some cases the correlation between the two fields is sufficient for quantitative relationships to be transferred. That two fields are distinguishable suggests that, in general, not all ideas of one are transferable to the other, otherwise they would be in complete equivalence. If two fields are not in a complete equivalence then it can not be assumed that a particular element (process or structure) of one will be found in the other, even though the surrounding details are similar in both. 
For example, a proof obtained in one field, using the elements contained within that field, has no $a$ priori value in another non-equivalent field except as a guide for investigation. A view of all the facts of chemistry (or physics) and computational (or communication) science would suggest that these two fields are not equivalent. Nor does one completely contain the other. Thus, to propose and consider proven [9] that a Maxwell's valve (Demon) in one of these fields is impossible does not infer that the equivalent mechanism in the other field is also disallowed.

Maxwell's Demon is of relevance to communication science because it provided an inspiration for that field's birth through the concern for intelligent choices. In the era before physicochemical explanations could be made for life-providing processes, the Demon in the natural sciences was a conundrum to which Szilard drew attention. Even Kelvin, the name-giver of Maxwell's Demon prefaced his statement of the Second Law with a condition that excluded the components of life. ("It is impossible, by means of inanimate material agency...." [10].) However, this period has now past and the term "Demon" should be for the physical and biological sciences only a poetic or historical reference to what must constitute a valve. This portrayal was preferred by its creator [11]. Thus, Maxwell's Demon should now lie in the realm of Computer Science or its equivalent and have no value in the natural sciences, where its counterpart, Maxwell's valve, is a construct for the testing of thermodynamic limits and the removal of the constraint of the second law.

\section{Chemical Kinetics}

If one were to make with real components, a machine of the type described in Fig. 3, the domain of chemical processes would be a likely environment in which to investigate. After all, the kinetic theory of chemical processes has been developed so that fluctuations are part of the founding constructs.

From quantum mechanical calculations it can be shown that when molecules undergo reactions, their energy first increases to a maximum and then decreases as the products are formed. This is the manner in which new bonds are formed between reacting species or, in simpler reactions, where new configurations are developed within a single species undergoing a conformational change.

If potential energy is lost when a molecule of $\mathrm{A}$ reconfigures to form $\mathrm{B}$ and the local temperature subsequently rises, then potential energy is gained at the expense of the local temperature 
when the reaction reverses. These processes are fluctuations that occur whether or not there is an observer. How can these fluctuations be utilized? Where lies the mystery of thermodynamically irreversible molecular events? Onsager [12] reviewed this problem over 70 years ago and recently, suggestions from that report have lead to a challenge of the second law where centrifugal forces were shown to influence differentially the equilibrium reaction rates [13]. In another contribution [14] this type of irreversibility was quantified. However, microscopic irreversibility can be achieved by methods [15] other than those considered by Onsager.

\section{References}

1. Szilard, L. Über die Entropieverminderung in einem thermodynischen System bei Eingriffin intelligenter Wesen. Z. f. Physik 1929, 53, 840-856. English Translation. Behavioral Science 1964, 9,301-310.

2. Popper, K. In The Philosophy of Karl Popper; Schilpp, P. A., Ed.; Open Court: Illinois, 1974; Vol. 1, Autobiography, pp 129-133.

3. Rothstein, J., In The Maximum Entropy Formalism, Levine, R. D.; Tribus, M., Eds.; MIT Press: Cambridge, Mass., 1979; pp 423-468.

4. Feynman, R.P.; Leighton, R. B.; Sands, M. The Feynman Lectures on Physics; Addison-Wesley: Reading, Mass., 1963; Vol. 1, Chapter 46, pp 1-9.

5. Zurek, W. H. In Frontiers of Nonequilibrium Statistical Physics; Moore, G. T.; Scully, M. O., Eds.; Plenum Press: New York, 1984; Maxwell's Demon, Szilard's Engine and Quantum Measurements, pp 151-161.

6. Einstein, A. In Albert Einstein: Philosopher-Scientist; Schilpp, P. A., Ed: Library of Living Philosophers Inc, Illinois, 1949; pp 47-49.

7. Perrin, J. La Loi de Stokes et le mouvement brownien. Comptes Rendus 1908, pp 465-6

8 Bohm, D. A suggested interpretation of the quantum theory in terms of "hidden" variables I and II. Phys. Rev. 1952, 85, 166 and 180.

9. Bennett, C. H. Demons, Engines and the second law. Sci. Am. 1987, 257, 88-96.

10. Thomson, W. In Mathematical and Physical Papers (vol 2), Cambridge Univ. Press, 1882, 511 
11. Maxwell, J. C. In Life and Scientific Work of Peter Guthrie Tait. Knott, C. G.; Cambridge Univ. Press, London, 1911; Letter to P. G. Tait.

12. Onsager, L. Reciprocal relations in irreversible processes. I. Phys. Rev. 1931, 37, 405-426.

13. Gordon, L. G. M. In Quantum limits to the second law; Sheehan, D. P.; AIP Conference Proc. San Diego, 2002; Vol. 643, pp 242-247.

14. Gordon, L. G. M. A Maxwellian valve based on centrifugal forces. Entropy 2004, this issue.

15. Gordon, L.G.M. Brownian Motion and Microscopic Irreversibility. Found. Phys. 1981, 11, 103-113

(C) 2004 by MDPI (http://www.mdpi.org). Reproduction for noncommercial purposes permitted. 